\title{
A Closed Form Evaluation of the Elliptic Integral
}

\author{
By M. L. Glasser and V. E. Wood
}

Abstract. The complete elliptic integral of the first kind $K(k)$ is evaluated in terms of gamma functions for the moduli $k=\sqrt{ } 2-1$ and $(2 \sqrt{ } 2-2)^{1 / 2}$.

We have found a new identity relating to the complete elliptic integral of the first kind $K(k)$ for modulus $k=\tan \pi / 8=\sqrt{ } 2-1$. By combining this with Special Case I of Abel's [1] theorem, we are able to express $K$ and $K^{\prime}$ of this modulus directly in terms of the gamma function. While such evaluations are interesting in themselves, they should also be useful for performing cross checks on the accuracy of numerical procedures.

The case $a=b=1$ of a result due to one of the authors [2] gives

$$
\begin{aligned}
I & =\int_{0}^{\infty} K\left[\frac{\left(x^{2}+1\right)^{1 / 2}-1}{\left(x^{2}+1\right)^{1 / 2}+1}\right] \frac{\left[\left(x^{2}+1\right)^{1 / 2}-1\right]^{1 / 2}}{\left(x^{2}+1\right)^{1 / 2}+1} \frac{d x}{\left(x^{2}+1\right)^{1 / 2}} \\
& =2(\sqrt{ } 2-1) K(\sqrt{ } 2-1) K^{\prime}(\sqrt{ } 2-1),
\end{aligned}
$$

where $K^{\prime}(k)=K\left(k^{\prime}\right)$ and $k^{\prime}=\left(1-k^{2}\right)^{1 / 2}$ is the complementary modulus. The substitution $k=\left[\left(x^{2}+1\right)^{1 / 2}-1\right] /\left[\left(x^{2}+1\right)^{1 / 2}+1\right]$ gives

$$
I=\frac{1}{\sqrt{ } 2} \int_{0}^{1}(1-k)^{-1 / 2} K(k) d k \text {. }
$$

By using the facts that

$$
K(k)=(\pi / 2){ }_{2} F_{1}\left(\frac{1}{2}, \frac{1}{2} ; 1 ; k^{2}\right) \text { and } \int_{0}^{1} k^{2 n}(1-k)^{-1 / 2} d k=B\left(\frac{1}{2}, 2 n+1\right),
$$

we find

$$
\begin{aligned}
I & =2^{-3 / 2} \pi \sum_{n=0}^{\infty} \frac{\left(\frac{1}{2}\right)_{n}\left(\frac{1}{2}\right)_{n}}{(1)_{n} n !} \frac{\Gamma(1 / 2) \Gamma(2 n+1)}{\Gamma(2 n+3 / 2)} \\
& =\frac{\pi^{2}}{4 \Gamma(3 / 4) \Gamma(5 / 4)} \sum_{n=0}^{\infty} \frac{\left(\frac{1}{2}\right)_{n}\left(\frac{1}{2}\right)_{n}\left(\frac{1}{2}\right)_{n}}{(3 / 4)_{n}(5 / 4)_{n} n !} .
\end{aligned}
$$

The duplication formula for the gamma function has been used to obtain the last expression in (3). The sum in (3) is simply ${ }_{3} F_{2}\left(\frac{1}{2}, \frac{1}{2}, \frac{1}{2} ; \frac{3}{4}, \frac{5}{4} ; 1\right)$ and by Whipple's theorem [3] we obtain

$$
I=\frac{\pi^{3}}{4[\Gamma(5 / 8) \Gamma(7 / 8)]^{2}}
$$

Received October 22, 1970.

AMS 1970 subject classifications. Primary 33A25; Secondary 33A30.

Key words and phrases. Complete elliptic integrals, generalized hypergeometric series.

Copyright $\odot 1971$, American Mathematical Society 
From (1) and (4) we have

$$
K(\sqrt{ } 2-1) K^{\prime}(\sqrt{ } 2-1)=\frac{\pi^{3}(\sqrt{ } 2+1)}{8[\Gamma(5 / 8) \Gamma(7 / 8)]^{2}} .
$$

We can now combine (5) with Abel's result

$$
K(\sqrt{ } 2-1) / K^{\prime}(\sqrt{ } 2-1)=1 / \sqrt{ } 2
$$

and we find

$$
\begin{aligned}
& K(\sqrt{ } 2-1)=\frac{\pi^{3 / 2}(2+\sqrt{ } 2)^{1 / 2}}{4 \Gamma(5 / 8) \Gamma(7 / 8)}=1.645568395 \cdots=K^{\prime}(2 \sqrt{ } 2-2)^{1 / 2}, \\
& K^{\prime}(\sqrt{ } 2-1)=\frac{\pi^{3 / 2}(4+2 \sqrt{ } 2)^{1 / 2}}{4 \Gamma(5 / 8) \Gamma(7 / 8)}=2.327185142 \cdots=K(2 \sqrt{ } 2-2)^{1 / 2} .
\end{aligned}
$$

To the authors' knowledge (6) and (7) together with Legendre's results $K(1 / \sqrt{ } 2)=$ $K^{\prime}(1 / \sqrt{ } 2)=\left[\Gamma\left(\frac{1}{4}\right)\right]^{2} / 4 \pi^{1 / 2}$ and $K[\sqrt{ } 2(\sqrt{ } 3-1) / 4]=\pi^{1 / 2} \Gamma\left(\frac{1}{6}\right) / 2 \cdot 3^{3 / 4} \Gamma\left(\frac{2}{3}\right)=$ $K^{\prime}[\sqrt{ } 2(\sqrt{ } 3-1) / 4] / \sqrt{ } 3$ appear to represent the only real cases where the elliptic integral has been expressed explicitly in terms of gamma functions (except for the trivial cases $K(0)=\pi / 2, K(1)=\infty)$. In the complex case only the value $K\left(e^{i x / 3}\right)=$ $e^{-i \pi / 3} \pi^{1 / 2} \Gamma\left(\frac{1}{6}\right) / 2\left(3^{3 / 4}\right) \Gamma\left(\frac{2}{3}\right)$ appears to be known. The list can be extended somewhat by use of Landen's transformation.

The identity (5) is rather mysterious, having been obtained outside the proper theory of elliptic functions, and it is an interesting question whether it is unique or hints at something deeper. The fact that $K(\sqrt{ } 2-1)$ should be expressible in terms of gamma functions is stated by Ramanujan [4], but he does not give any details.

Battelle Memorial Institute

505 King Avenue

Columbus, Ohio 43201

1. E. T. Whittaker \& G. N. Watson, A Course of Modern Analysis, 4th ed., Cambridge Univ. Press, New York, 1962, sect. 22.81. MR 31 \#2375.

2. M. L. GlasSER, "An elliptic integral identity," Math. Comp., v. 25, 1971, pp. 533-534.

3. W. N. BaIley, Generalized Hypergeometric Series, Cambridge Univ. Press, Cambridge, 1935.

4. S. Ramanujan, "Modular equations and approximations to $\pi$," Quart. J. Math., v. 45, 1914 , pp. $350-372$, sect. 15 . 